\title{
Transmit Power Allocation for BER Performance Improvement in Multicarrier Systems
}

\author{
Chang Soon Park, Student Member, IEEE, and Kwang Bok Lee, Senior Member, IEEE
}

\begin{abstract}
In a multicarrier system, transmit power allocation over different subchannels is an effective means of improving the performance. We develop the optimal transmit power allocation scheme to improve bit-error rate (BER) performance in a multicarrier system with diversity reception. A simple suboptimal scheme is also derived from the optimal one, and an asymptotic case referred to as the equal-signal-to-noise ratio scheme is discussed. Numerical results show that the optimal and suboptimal power allocation schemes significantly outperform the equal power allocation scheme. The effects of the modulation level, the number of receiving antennas, and the number of subchannels on the BER performance are also investigated.
\end{abstract}

Index Terms-Bit-error rate (BER), diversity, multicarrier system, transmit power allocation.

\section{INTRODUCTION}

$\mathbf{M}$ ULTICARRIER communication systems are promising for future wideband wireless communications, and recently the system is being applied to several fixed and mobile radio systems, such as digital audio and video broadcasting, and wireless LAN [1], [2]. In a multicarrier system, a wideband channel is divided into multiple narrowband subchannels using orthogonal subcarriers. Multiple data substreams are transmitted in parallel through subchannels, and the total transmit power should be distributed to these subchannels. It may be natural to allocate equal transmit power to multiple subchannels, when the channel state information (CSI) is not available at the transmitter. When the CSI is available at the transmitter, however, effective transmit power allocation may improve error-rate performance or increase the capacity. It has been well known that the optimal transmit power allocation for capacity maximization is the waterfilling power allocation [3], [4]. Various bit-loading algorithms based on the waterfilling strategy have been investigated in [5]-[8]. These schemes of increasing capacity may be suitable for variable-rate services, such as e-mail and web browsing. On the contrary, delay-sensitive services, such as voice or video, are usually provided at a fixed rate [9]. In these applications, it is desirable to design a transmit power allocation scheme that improves error-rate performance for a given rate. Although the error-rate performance of power allocation in multicarrier systems has been studied in a few papers [10], [11], the optimal power allocation for

Paper approved by V. A. Aalo, the Editor for Diversity and Fading Channel Theory of the IEEE Communications Society. Manuscript received April 30, 2002; revised October 1, 2003 and February 25, 2004. This paper was presented in part at the IEEE International Symposium on Personal, Indoor, and Mobile Radio Communications, Lisbon, Portugal, September 2002.

The authors are with the School of Electrical Engineering and Computer Science, Seoul National University, Seoul 151-742, Korea (e-mail: parkcs@mobile.snu.ac.kr; klee@snu.ac.kr)

Digital Object Identifier 10.1109/TCOMM.2004.836491 error-rate minimization and its characteristics have not been fully investigated yet.

We develop the optimal transmit power allocation scheme to improve bit-error rate (BER) performance in a multicarrier system with receive antenna diversity. Receive antenna diversity is used to mitigate the effects of fading, as in [12]. Based on the optimal scheme, a computationally efficient suboptimal scheme is also derived. Furthermore, it is shown that the equal signal-to-noise ratio (SNR) scheme, which makes the received SNR become the same for all subchannels, corresponds to an asymptotic case of the suboptimal scheme. The performance of the proposed power allocation schemes is evaluated, and compared with that of the equal power allocation scheme. The effects of the modulation level, the number of receiving antennas, and the number of subchannels on the BER performance are also investigated.

\section{System AND ChanNel Models}

A multicarrier communication system considered in this paper is depicted in Fig. 1. An input data stream is divided into $K$ parallel substreams through a serial-to-parallel converter. The transmit power $p_{k}$ is assigned to the $k$ th substream $d_{k}$ $(k=1,2, \ldots, K)$. In the multicarrier modulator, the substreams are modulated on orthogonal subcarriers to form a transmit signal. The receiver is equipped with $N$ antennas, from which $N$ replicas of the transmit signal are received. The multicarrier demodulator at each antenna separates the received signal into $K$ subcarrier signals. The signals received from different antennas are assumed to undergo independent fading. We also assume that the $K$ subcarrier signals for each antenna experience frequency-nonselective and slow fading independently. Correspondingly, the output of a multicarrier demodulator for the $k$ th subcarrier at the $n$th receive antenna may be expressed as

$$
y_{k, n}=\sqrt{p_{k}} h_{k, n} d_{k}+n_{k, n}, \quad k=1,2, \ldots, K ; n=1,2, \ldots, N
$$

where $h_{k, n}$ denotes the multiplicative fading coefficient for the $k$ th subcarrier at the $n$th antenna, and they are assumed to be independent and identically distributed (i.i.d.) circularly symmetric complex Gaussian random variables with zero mean and unit variance. $n_{k, n}$ 's represent the additive noise, and they are assumed to be i.i.d. circularly symmetric complex Gaussian random variables with zero mean and variance of $\sigma^{2} . d_{k}$ is the encoded data symbol with unit average power for the $k$ th subcarrier. It is assumed that the modulation levels, and hence, the bit rates are equal for all subcarriers. $p_{k}$ is the transmit 


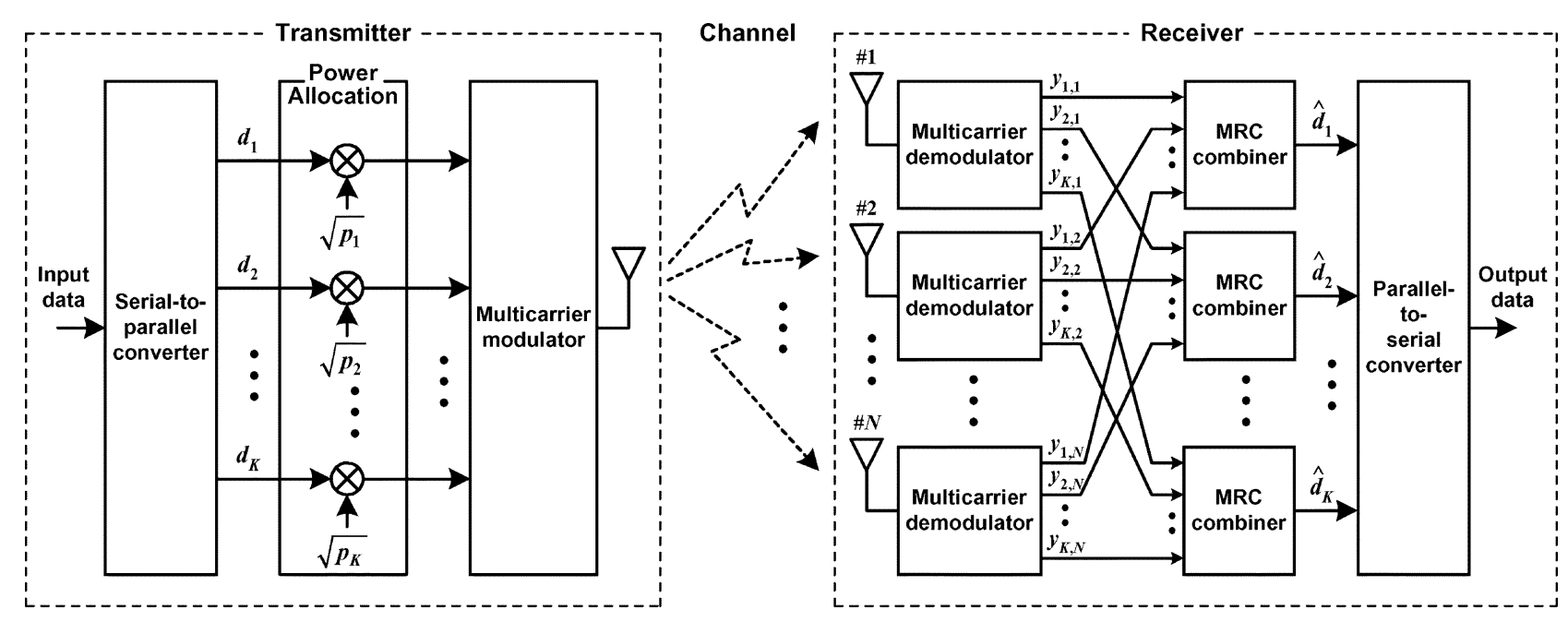

Fig. 1. Multicarrier communication system with receive antenna diversity.

power for the $k$ th subcarrier, with the total power constraint given as

$$
\sum_{k=1}^{K} p_{k}=K \bar{P}
$$

where $\bar{P}$ denotes the average transmit power per subcarrier, when the total transmit power is equally distributed into the $K$ subcarriers.

The signals received from $N$ antennas are combined for each subcarrier to achieve antenna gain and diversity gain. It is assumed that the maximal ratio combining (MRC) is employed to maximize the SNR [1]. The transmit symbol for each subcarrier is estimated based on the output of each MRC combiner. After $\mathrm{MRC}$, the SNR $\gamma_{k}$ for the $k$ th subcarrier is calculated as

$$
\gamma_{k}=\alpha_{k} p_{k}
$$

where $\alpha_{k} \triangleq \sum_{n=1}^{N}\left|h_{k, n}\right|^{2} / \sigma^{2}$ is the ratio of the combined gain of the $k$ th subchannel to the noise power, representing the overall channel state for the $k$ th subchannel. The channel states $\alpha_{k}$ 's required to determine the transmit powers $p_{k}$ 's are assumed to be perfectly known to the transmitter. These channel states can be obtained by feedback from the receiver in a frequency-division duplex system, or can be estimated at the transmitter in a time-division duplex system.

\section{TRAnSMit Power Allocation}

In this section, several effective transmit power allocation schemes are described. The power allocation scheme that is optimal in terms of the BER is developed in Section III-A. A suboptimal scheme is derived as a simplified version of the optimal scheme in Section III-B, and the equal SNR scheme is discussed as an asymptotic case of the suboptimal scheme in Section III-C.

\section{A. Optimal Power Allocation}

To derive the optimal power allocation scheme, we first express the overall BER as a function of the transmit power for $K$ subcarriers, $\left\{p_{k} \mid k=1,2, \ldots, K\right\}$, and then find $\left\{p_{k}\right\}$ that minimizes the overall BER. The BER for the $k$ th subcarrier is generally a function of the SNR $\gamma_{k}$, and thus, the BER $P_{b}\left(e \mid \alpha_{k}\right)$ for a given channel state $\alpha_{k}$ may be expressed as

$$
P_{b}\left(e \mid \alpha_{k}\right)=f\left(\gamma_{k}\right)=f\left(\alpha_{k} p_{k}\right), \quad k=1,2, \ldots, K
$$

where $f(\cdot)$ is a function determined by a specific modulation scheme. Since data streams are transmitted over independent subchannels with equal rate constraint, the overall BER for given channel states of $\left\{\alpha_{k} \mid k=1,2, \ldots, K\right\}$ can be calculated as an arithmetic mean of $P_{b}\left(e \mid \alpha_{k}\right)$

$P_{b}\left(e \mid \alpha_{1}, \alpha_{2}, \ldots, \alpha_{K}\right)=\frac{1}{K} \sum_{k=1}^{K} P_{b}\left(e \mid \alpha_{k}\right)=\frac{1}{K} \sum_{k=1}^{K} f\left(\alpha_{k} p_{k}\right)$.

Note that the average BER becomes minimal when the BER in (5) is minimized for each given channel state. To find the optimal $\left\{p_{k}\right\}$ that minimizes (5), we use the Lagrange multiplier method with the total power constraint in (2). The Lagrangian function may be expressed as

$$
J\left(p_{1}, p_{2}, \ldots, p_{K}\right)=\frac{1}{K} \sum_{k=1}^{K} f\left(\alpha_{k} p_{k}\right)+\lambda\left(\sum_{k=1}^{K} p_{k}-K \bar{P}\right)
$$

where $\lambda$ denotes the Lagrange multiplier. By differentiating (6) with respect to $p_{k}$ and setting it to zero, we obtain a set of $K$ equations as

$$
\frac{1}{K} \frac{d}{d p_{k}} f\left(\alpha_{k} p_{k}\right)+\lambda=0, \quad k=1,2, \ldots, K .
$$

Solving $K+1$ simultaneous equations in (2) and (7), we can calculate the optimal set of the transmit power $\left\{p_{k}\right\}$.

As mentioned above, the BER function in (4) is a function determined by a specific modulation scheme. For a binary differential phase-shift keying (DPSK) with single-antenna reception, for example, the BER function may be expressed as an exponential function [1], and a closed-form solution of (2) and (7) may be easily found. For an $M$-ary phase-shift keying (PSK) or $M$-ary quadrature amplitude modulation (QAM), however, the exact or approximate BER function may be expressed as a $Q$-function [1], and it may be difficult to find a closed-form solution. In this case, an adaptive method, such 
as the steepest-descent algorithm [13], may be employed to find a solution in an iterative manner as follows.

Stage 1) Initialization: Set an iteration number $i=0$, a step size $\mu(0)=\mu_{0}$, and an arbitrary initial positive power set $\left\{p_{k}(0)\right\}$ satisfying (2).

Stage 2) Power set update: For $k=1,2, \ldots, K$, update the transmit power $p_{k}(i)$ as

$$
\begin{aligned}
p_{k}(i+1) & =p_{k}(i)-\mu(i) \frac{\partial}{\partial p_{k}(i)} J\left(p_{1}(i), p_{2}(i), \ldots, p_{K}(i)\right) \\
& =p_{k}(i)-\mu(i)\left(\frac{1}{K} \frac{d}{d p_{k}(i)} f\left(\alpha_{k} p_{k}(i)\right)+\lambda(i)\right)
\end{aligned}
$$

where $\lambda(i)$ is determined from the power constraint in (2) and is updated as

$$
\lambda(i)=-\frac{1}{K^{2}} \sum_{k=1}^{K} \frac{d}{d p_{k}(i)} f\left(\alpha_{k} p_{k}(i)\right)
$$

Stage 3) Step-size adjustment: If all components of the updated power set $\left\{p_{k}(i+1)\right\}$ in Stage 2 are positive, then go to Stage 4 with $\mu(i+1)=\mu_{0}$. Otherwise, compute $\tilde{\mu}_{k}(i) \triangleq$ $p_{k}(i) /\left((1 / K)\left(d f\left(\alpha_{k} p_{k}(i)\right) /\left(d p_{k}(i)\right)+\lambda(i)\right)\right.$ for $k$ 's associated with $p_{k}(i+1) \leq 0$, set the step size $\mu(i)$ to $\rho \cdot \min _{k: p_{k}(i+1) \leq 0} \tilde{\mu}_{k}(i)$, where $\rho$ is a positive scaling factor smaller than one, ${ }^{1}$ and return to Stage 2 .

Stage 4) Repetition or termination: If more iterations are required for convergence, increase $i$ by one and go to Stage 2. Otherwise, terminate the adaptive procedure.

The adaptive algorithm described above converges to the global optimum solution for the convex BER function [14]. Note that the $Q$-function, which is the exact or approximate BER function for an $M$-ary PSK or $M$-ary QAM, is a convex function.

It is worthwhile to note that the characteristics of the optimal transmit power allocation scheme derived above differ from those of the waterfilling scheme that maximizes the capacity. As shown in the Appendix for the case of two subchannels, at high SNR range, the optimal power allocation scheme tends to allocate more transmit power to the more attenuated subchannel, which is contrary to the behavior of the waterfilling scheme.

\section{B. Suboptimal Power Allocation}

An approach simpler than the adaptive method in Section III-A is to find an approximate closed-form solution using a simple approximation of the BER rather than the exact BER expression. Based on this approach, in this subsection, we derive a closed-form transmit power allocation scheme for $M$-ary square $\left(M=4^{m}, m=1,2, \ldots\right)$ QAM schemes. For

\footnotetext{
${ }^{1}$ This step-size adjustment is performed to make all the updated power components become positive, and $\rho$ is set to 0.9 for numerical results in Section IV.
}

these schemes, the BER function in (4) may be approximated using an upper bound as [1], [15]

$$
f\left(\alpha_{k} p_{k}\right) \cong a Q\left(\sqrt{b \alpha_{k} p_{k}}\right) \leq \frac{a}{2} \exp \left(-\frac{b}{2} \alpha_{k} p_{k}\right)
$$

where $Q(x) \triangleq(1 / \sqrt{2 \pi}) \int_{x}^{\infty} \exp \left(-t^{2} / 2\right) d t$ denotes the $Q$-function, $a=2(\sqrt{M}-1) / \sqrt{M} \log _{2} \sqrt{M}$, and $b=3 /(M-1)$. By substituting the upper bound in (10) into the BER function of (7), we can find a closed-form solution of $K+1$ simultaneous equations in (2) and (7). Some components of the calculated power set may be negative. In this case, we apply the Kuhn-Tucker conditions [14], from which the negative components of $\left\{p_{k}\right\}$ are set to zero and the remaining components are recalculated until all components become nonnegative. Consequently, the solution is found as

$$
p_{k}= \begin{cases}\frac{\lambda_{0}}{\alpha_{k}}-\left(\frac{2}{b}\right)\left(\frac{1}{\alpha_{k}}\right) \ln \left(\frac{1}{\alpha_{k}}\right), & \alpha_{k} \geq \exp \left(-\frac{b \lambda_{0}}{2}\right) \\ 0, & \alpha_{k}<\exp \left(-\frac{b \lambda_{0}}{2}\right)\end{cases}
$$

where $\lambda_{0} \triangleq-(2 / b) \ln (4 K \lambda / a b)$ is calculated to satisfy the power constraint in (2) as

$$
\lambda_{0}=\frac{K \bar{P}+\left(\frac{2}{b}\right) \sum_{k \in S}\left(\frac{1}{\alpha_{k}}\right) \ln \left(\frac{1}{\alpha_{k}}\right)}{\sum_{k \in S}\left(\frac{1}{\alpha_{k}}\right)}
$$

where $S=\left\{k \mid \alpha_{k} \geq \exp \left(-b \lambda_{0} / 2\right)\right\}$. From (11), it can be seen that no transmit power is allocated to the $k$ th subcarrier if the channel state $\alpha_{k}$ is smaller than $\exp \left(-b \lambda_{0} / 2\right)$. Furthermore, it can be easily shown that a positive transmit power $p_{k}$ in (11) increases with $\alpha_{k}$ for $\exp \left(-b \lambda_{0} / 2\right)<\alpha_{k} \leq \exp \left(1-b \lambda_{0} / 2\right)$, while $p_{k}$ decreases with $\alpha_{k}$ for $\alpha_{k}>\exp \left(1-b \lambda_{0} / 2\right)$.

\section{Equal SNR Power Allocation}

The transmit power can be assigned to subcarriers so that the received SNR $\Upsilon_{k}$ in (3), and hence, the BER $f\left(\Upsilon_{k}\right)$ in (4) become equal for all subchannels. We call this scheme the equal SNR power allocation scheme. It can be shown that the equal SNR power allocation scheme corresponds to an asymptotic case of the suboptimal scheme in (11). From (11) and (12), it can be shown that $p_{k}$ becomes $\lambda_{0} / \alpha_{k}$ asymptotically, when $\alpha_{k}$ is sufficiently large for all $k$. In this case, the power allocation in (11) may be written as

$$
p_{k}=\frac{\lambda_{0}}{\alpha_{k}}=\frac{K \bar{P}}{\sum_{\ell=1}^{K}\left(\frac{1}{\alpha_{\ell}}\right)} \cdot \frac{1}{\alpha_{k}} .
$$

The corresponding SNR is calculated as

$$
\gamma_{k}=\lambda_{0}=\frac{K \bar{P}}{\sum_{\ell=1}^{K}\left(\frac{1}{\alpha_{\ell}}\right)}, \quad k=1,2, \ldots, K
$$

which indicates that the received SNR becomes equal for all subchannels by the power allocation in (13). Consequently, it has been shown that the suboptimal scheme of (11) behaves like the equal SNR scheme asymptotically, when all the subchannels are in sufficiently good condition. The equal SNR scheme allocates transmit power inversely proportional to the channel state $\alpha_{k}$, allocating more transmit power to the more attenuated subchannel. 


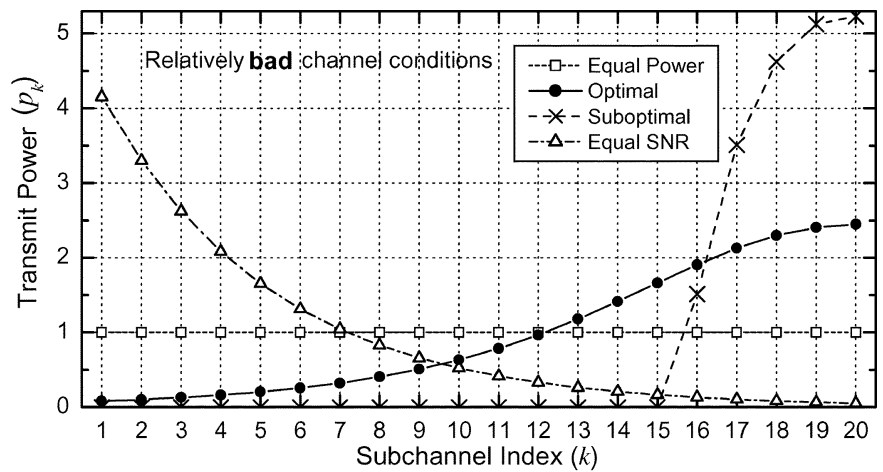

(a)

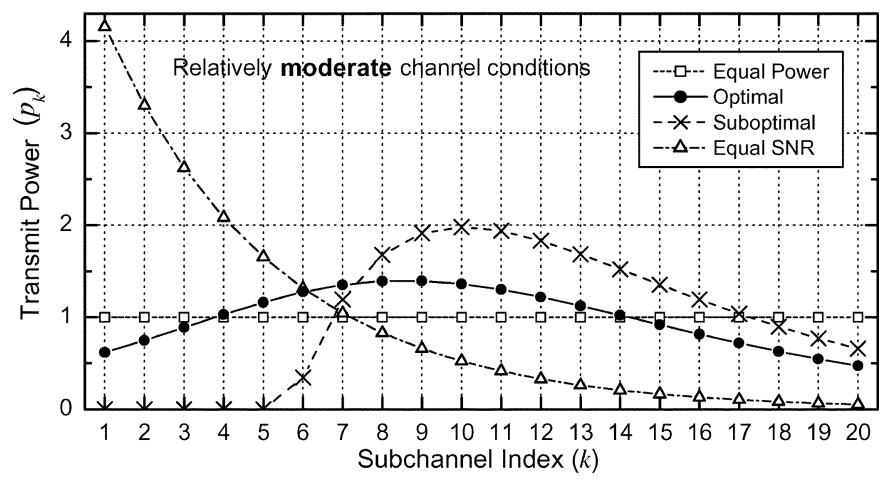

(b)

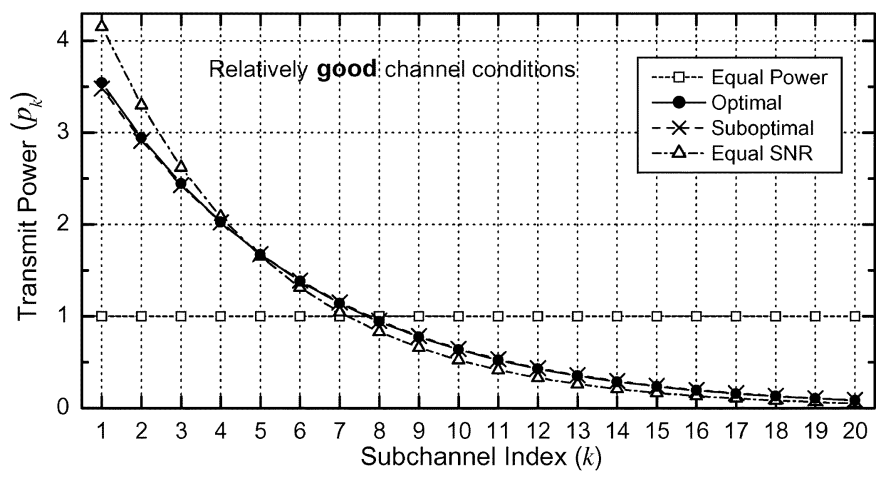

(c)

Fig. 2. Characteristics of power allocation schemes for $M=4, K=20$, and $\bar{P}=1$. (a) $\alpha_{k}=-24+k \mathrm{~dB}$. (b) $\alpha_{k}=-10+k \mathrm{~dB}$. (c) $\alpha_{k}=3+k \mathrm{~dB}$.

\section{NUMERICAL RESULTS}

In this section, the performance of the transmit power allocation schemes described in Section III are evaluated and compared with one another. A QAM is assumed to be employed for each subcarrier. The average SNR is defined to be $\bar{P} / \sigma^{2}$. The average BER for each transmit power allocation scheme is calculated by averaging the BER in (5) over sufficient number of randomly generated channel states $\alpha_{k}(k=1,2, \ldots, K)$.

Prior to the average BER performance comparisons, the characteristics of power allocation schemes are illustrated in Fig. 2(a)-(c), which show the transmit power $p_{k}$ calculated from four power allocation schemes for specific values of $\alpha_{k}$, when the number of subcarriers $K=20$, modulation level $M=4$, and $\bar{P}=1$. The channel states $\alpha_{k}$ 's are set to equispaced values in a given range: $[-23 \mathrm{~dB},-4 \mathrm{~dB}]$ in Fig. $2(\mathrm{a})$, [-9 dB, $10 \mathrm{~dB}]$ in Fig. 2(b), and [4 dB, $23 \mathrm{~dB}]$ in Fig. 2(c). These ranges of $\alpha_{k}$ in Fig. 2(a)-(c), respectively, stand for

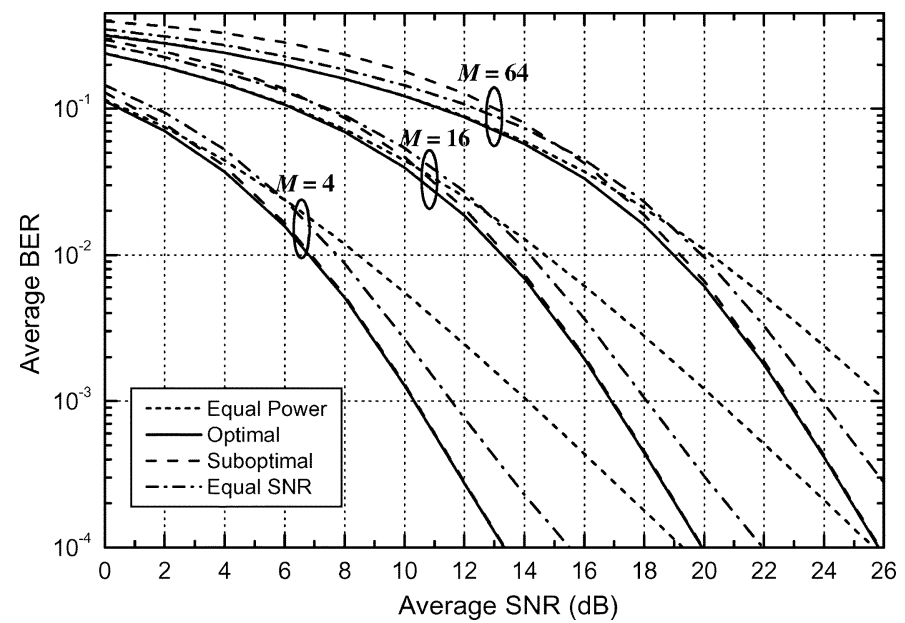

Fig. 3. BER performance comparisons of power allocation schemes for various modulation levels $M$, when $K=8$ and $N=2$.

relatively bad, moderate, and good channel conditions. The optimal power allocation scheme is observed to assign more transmit power to a subchannel with larger $\alpha_{k}$, or to a less attenuated subchannel, in Fig. 2(a), while the reverse trend is observed in Fig. 2(c). This indicates that the optimal power allocation behaves like "waterfilling" in bad channel conditions, whereas it behaves like "inverse waterfilling" in good channel conditions. A mixture of these two behaviors can be shown in Fig. 2(b). The trends of the suboptimal power allocation scheme are shown to be similar to that of the optimal power allocation scheme, except that the power corresponding to some highly attenuated subchannels is forced to zero, according to (11). As expected, the equal SNR scheme allocates more transmit power to the more attenuated subchannel for all cases. It can be seen from Fig. 2(c) that the equal SNR scheme approaches the optimal or suboptimal scheme, when all the subchannels are in sufficiently good condition.

Fig. 3 compares the average BER performance of the transmit power allocation schemes for various modulation levels $M$, when $K=8$ and $N=2$. As expected, the optimal power allocation scheme provides the best performance for all cases. It is noticeable that the performance of the optimal and suboptimal schemes is almost indistinguishable at high SNR range. However, the performance difference between these two schemes increases, as SNR decreases and/or $M$ increases. The reason for this is that the approximate BER in (10) used for deriving the suboptimal scheme becomes more inaccurate, as SNR decreases and/or $M$ increases. The performance improvement of the optimal and suboptimal schemes over the other schemes is seen to be larger for lower modulation levels. In the case of quaternary phase-shift keying (QPSK) modulation $(M=4)$, the SNR gain of the optimal scheme is about $3.8 \mathrm{~dB}$ over the equal power scheme, and $1.3 \mathrm{~dB}$ over the equal SNR scheme at BER of $10^{-3}$.

Fig. 3 also shows that the equal SNR scheme is superior to the equal power scheme at high SNR range, while it is inferior to the equal power scheme at low SNR range. This may be explained using Fig. 2. As shown in Fig. 2, the equal SNR scheme is close to the optimal scheme, when all the subchannels are in good condition. When all the subchannels are in bad condition, however, the equal SNR scheme tends to allocate transmit 


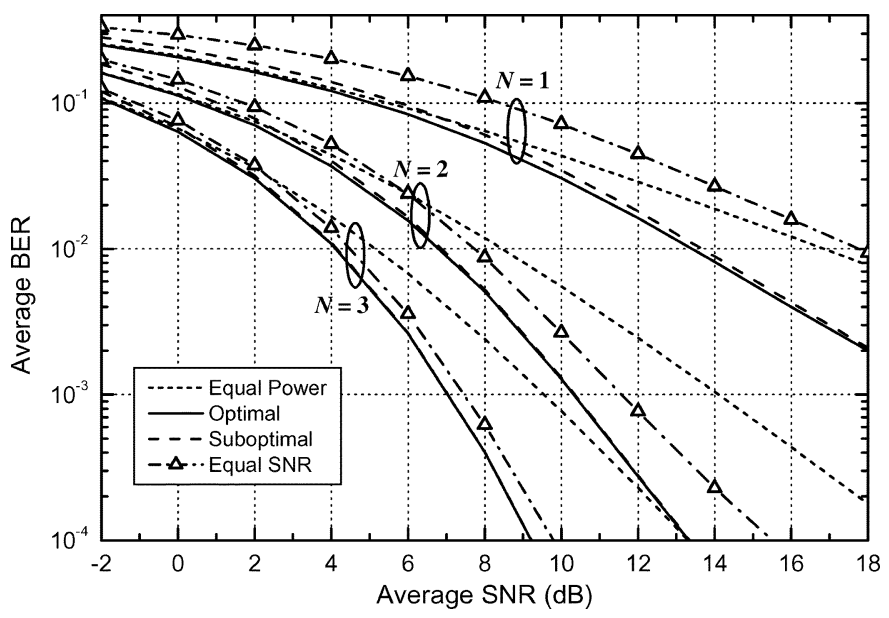

Fig. 4. Effects of the number of receiving antennas $N$ on the BER performance of power allocation schemes, when $M=4$ and $K=8$.

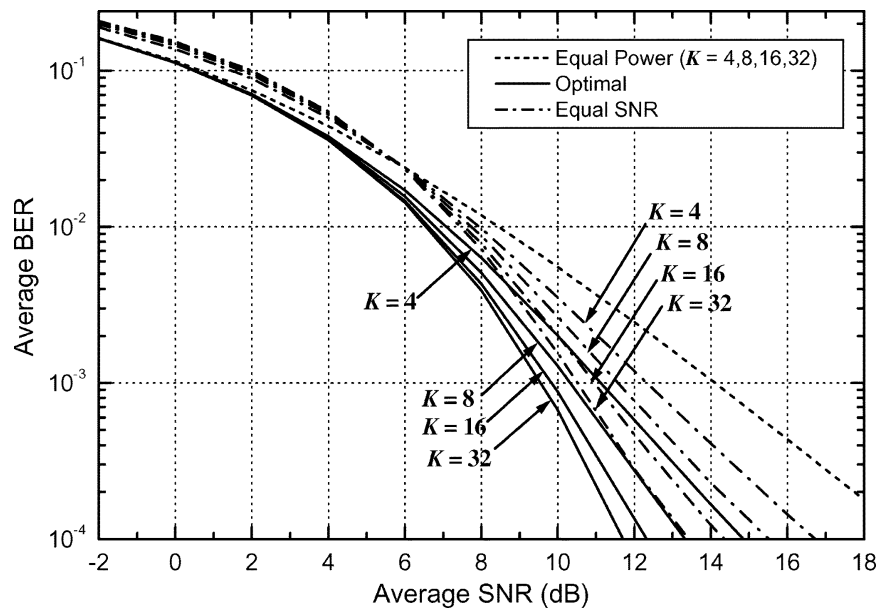

Fig. 5. Effects of the number of subchannels $K$ on the BER performance of power allocation schemes, when $M=4$ and $N=2$.

power in a fashion contrary to the optimal scheme. In this case, the equal power scheme is closer to the optimal scheme than the equal SNR scheme is.

The effects of the number of receiving antennas $N$ on the BER performance are shown in Fig. 4 , when $M=4$ and $K=8$. As shown in Fig. 3, the optimal and suboptimal schemes significantly outperform the equal power scheme, for any value of $N$. We can observe that the equal SNR scheme also outperforms the equal power scheme, unless the SNR is very low or the number of receiving antennas is small. Furthermore, the performance of the equal SNR scheme is found to approach that of the optimal or suboptimal scheme, as $N$ increases. When $N=3$, the performance difference is as small as $0.5 \mathrm{~dB}$ at BER of $10^{-3}$. This implies that increased "diversity effects" resulting from more receiving antennas provide a higher probability of all the subchannels being in sufficiently good condition. Hence, the simple equal SNR scheme can be an alternative to the optimal or suboptimal scheme, when the number of receiving antennas is sufficiently large.

Fig. 5 shows the effects of the number of subchannels $K$ on the BER performance, when $M=4$ and $N=2$. Note that the performance of the equal power scheme is independent of $K$. The SNR gains of the optimal and equal SNR schemes over the equal power scheme are found to increase with $K$ increasing. Note that greater $K$ also makes the BER curves for the optimal and equal SNR schemes decline more rapidly with SNR increasing. This phenomenon indicates that the optimal and equal SNR power allocation schemes can achieve additional diversity effects from the increased number of subchannels.

\section{CONCLUSIONS}

We have developed the optimal transmit power allocation scheme that improves BER performance in a multicarrier system with diversity reception. A computationally efficient suboptimal scheme has also been derived for $M$-ary QAM, and the equal SNR scheme has been shown to be an asymptotic case of the suboptimal scheme. The BER performance improvement of the optimal scheme over the equal power scheme has been found to increase, as the number of subchannels increases. The suboptimal scheme has been shown to perform as well as the optimal scheme at high SNR range. It has also been found that the performance of the equal SNR scheme approaches that of the suboptimal scheme, as the number of receiving antennas (i.e., antenna diversity gain) increases.

\section{APPENDIX}

In this appendix, we compare the characteristics of the optimal power allocation scheme derived in Section III-A and those of the waterfilling power allocation for the case of two subchannels. Note that the objective of the proposed scheme is to minimize the overall BER, while that of the waterfilling scheme is to maximize the overall capacity, under the total power constraint. We will show that different properties of the "derivatives" for the BER and capacity functions lead to the different characteristics of the power allocation schemes.

The Lagrangian funtion for the two power allocation schemes may be expressed as

$$
J\left(p_{1}, p_{2}\right)=F\left(p_{1}, p_{2}\right)+\lambda\left(\sum_{k=1}^{2} p_{k}-2 \bar{P}\right)
$$

where $F\left(p_{1}, p_{2}\right)=(1 / 2) \sum_{k=1}^{2} Q\left(\sqrt{\alpha_{k} p_{k}}\right)$ in the case of the proposed scheme with QPSK modulation, and $F\left(p_{1}, p_{2}\right)=\sum_{k=1}^{2} \log _{2}\left(1+\alpha_{k} p_{k}\right)$ in the case of the waterfilling scheme [3]. Differentiating (15) with respect to $p_{k}$ and setting it to zero, we obtain

$$
D\left(\alpha_{k}, p_{k}\right)=-\lambda, \quad k=1,2
$$

where

$$
\begin{aligned}
D\left(\alpha_{k}, p_{k}\right) & =-\frac{1}{4 \sqrt{2 \pi}} \sqrt{\frac{\alpha_{k}}{p_{k}}} \exp \left(-\frac{1}{2} \alpha_{k} p_{k}\right) \\
& \triangleq D_{\mathrm{BER}}\left(\alpha_{k}, p_{k}\right)
\end{aligned}
$$

in the case of the proposed scheme, and $D\left(\alpha_{k}, p_{k}\right)=$ $(1 / \ln 2) /\left(p_{k}+1 / \alpha_{k}\right) \triangleq D_{\text {cap }}\left(\alpha_{k}, p_{k}\right)$ in the case of the waterfilling scheme. We observe the following differences between the two derivatives.

1) $D_{\mathrm{BER}}\left(\alpha_{k}, p_{k}\right)$ is a monotonically increasing function of the positive $p_{k}$, whereas $D_{\text {cap }}\left(\alpha_{k}, p_{k}\right)$ is a monotonically decreasing function of $p_{k}$. 
2) $D_{\mathrm{BER}}\left(\alpha_{k}, p_{k}\right)$ for $p_{k}=\bar{P}$ shows opposite trends, according to the range of $\alpha_{k}$

$$
\begin{cases}D_{\mathrm{BER}}\left(\alpha_{1}, \bar{P}\right)>D_{\mathrm{BER}}\left(\alpha_{2}, \bar{P}\right), & \text { if } \alpha_{1}<\alpha_{2} \leq \frac{1}{\bar{P}} \\ D_{\mathrm{BER}}\left(\alpha_{1}, \bar{P}\right)<D_{\mathrm{BER}}\left(\alpha_{2}, \bar{P}\right), & \text { if } \frac{1}{\bar{P}}<\alpha_{1}<\alpha_{2}\end{cases}
$$

On the other hand, $D_{\text {cap }}\left(\alpha_{k}, p_{k}\right)$ shows a consistent trend for all the range of $\alpha_{k}$

$$
D_{\text {cap }}\left(\alpha_{1}, \bar{P}\right)<D_{\text {cap }}\left(\alpha_{2}, \bar{P}\right), \quad \text { if } \alpha_{1}<\alpha_{2} .
$$

Note that the optimal powers $p_{k}^{*}$ 's should make $D\left(\alpha_{k}, p_{k}\right)$ become equal for two subchannels, as in (16). From the above observations on $D_{\mathrm{BER}}\left(\alpha_{k}, p_{k}\right)$, it can be easily shown that, for some positive value $\Delta, p_{1}^{*}=\bar{P}-\Delta$ and $p_{2}^{*}=\bar{P}+\Delta$ if $\alpha_{1}<\alpha_{2} \leq 1 / \bar{P}$, while $p_{1}^{*}=\bar{P}+\Delta$ and $p_{2}^{*}=\bar{P}-\Delta$ if $1 / \bar{P}<\alpha_{1}<\alpha_{2}$. This indicates that more transmit power should be allocated to the subchannel with larger $\alpha_{k}$ (smaller $\left.\alpha_{k}\right)$ in a range of $\alpha_{k} \leq 1 / \bar{P}\left(\alpha_{k}>1 / \bar{P}\right)$. This result is consistent with an intuitive view, since it may be effective to allocate more power to subchannel with a "steeper" BER slope, or smaller $D_{\mathrm{BER}}\left(\alpha_{k}, p_{k}\right)$, to reduce the overall BER. On the other hand, from the observations on $D_{\text {cap }}\left(\alpha_{k}, p_{k}\right)$, it can be seen that more power should be allocated to the subchannel with larger $\alpha_{k}$ for all the range of $\alpha_{k}$, which is the well-known behavior of the waterfilling scheme.

\section{REFERENCES}

[1] J. G. Proakis, Digital Communications. New York: McGraw-Hill, 1995.

[2] J. A. C. Bingham, "Multicarrier modulation for data transmission: An idea whose time has come," IEEE Commun. Mag., vol. 28, pp. 5-14, May 1990.
[3] T. M. Cover and J. A. Thomas, Elements of Information Theory. New York: Wiley, 1991.

[4] J. Jang, K. B. Lee, and Y.-H. Lee, "Frequency-time domain transmit power adaptation for a multicarrier system in fading channels," in Proc. IEEE Int. Symp. Personal, Indoor, and Mobile Radio Communications, San Diego, CA, Sept.-Oct. 2001, pp. D100-D103.

[5] S. T. Chung and A. J. Goldsmith, "Adaptive multicarrier modulation for wireless systems," in Proc. 34th Asilomar Conf. Signals, Systems, Computers, vol. 2, 2000, pp. 1603-1607.

[6] P. S. Chow, J. M. Cioffi, and J. A. C. Bingham, "A practical discrete multitone transceiver loading algorithm for data transmission over spectrally shaped channels," IEEE Trans. Commun., vol. 43, pp. 773-775, Feb.-Apr. 1995.

[7] B. S. Krongold, K. Ramchandran, and D. L. Jones, "Computationally efficient optimal power allocation algorithms for multicarrier communication systems," IEEE Trans. Commun., vol. 48, pp. 23-27, Jan. 2000.

[8] R. V. Sonalkar and R. R. Shively, "An efficient bit-loading algorithm for DMT applications," IEEE Commun. Lett., vol. 4, pp. 80-82, Mar. 2000.

[9] "QoS Concept and Architecture," 3rd Generation Partnership Project (3GPP), Tech. Spec. 23.107, V.4.0.0, 2000.

[10] H.-J. Su and E. Geraniotis, "Power allocation and control for multicarrier systems with soft decoding," IEEE J. Select. Areas Commun., vol. 17, pp. 1759-1769, Oct. 1999.

[11] R. F. H. Fischer and J. B. Huber, "A new loading algorithm for discrete multitone transmission," in Proc. GLOBECOM, London, U.K., Nov. 1996, pp. 724-728.

[12] A. A. Hutter, J. S. Hammerschmidt, E. Carvalho, and J. M. Cioffi, "Receive diversity for mobile OFDM systems," in Proc. IEEE WCNC, Chicago, IL, Sept. 2000, pp. 707-712.

[13] B. Widrow and S. D. Stearns, Adaptive Signal Processing. Englewood Cliffs, NJ: Prentice-Hall, 1985.

[14] D. G. Luenberger, Introduction to Linear and Nonlinear Programming. Reading, MA: Addison-Wesley, 1973.

[15] J. M. Wozencraft and I. M. Jacobs, Principles of Communication Engineering. New York: Wiley, 1965. 